\title{
Autosomal recessive frontotemporal pachygyria
}

INSERM

\section{Source}

INSERM. (1999). Orphanet: an online rare disease and orphan drug data base. Autosomal recessive frontotemporal pachygyria. ORPHA:329329

Autosomal recessive frontotemporal pachygyria is a cerebral malformation characterized by symmetric, bilateral pachygyria with normal head circumference and without polymicrogyria. Clinical manifestations include developmental delay, moderate intellectual disability, normal or slightly decreased muscle tone and deep-tendon reflexes, telecanthus or hypertelorism. 